\title{
Monte Carlo Simulation of Muscarine in Water Solution
}

\author{
Chiara Margheritis \\ Department of Inorganic Chemistry, University of Messina, Italy
}

Z. Naturforsch. 45a, 1009-1015 (1990); received May 29, 1990

\begin{abstract}
Monte Carlo simulations of muscarine water solution were carried out using ab-initio SCFLCAO-MO interaction potentials. The overall results indicate that in water solution the preferred conformation, on the basis of enthalpic considerations, should be the one having the dihedral angle $\mathrm{HO} 2-\mathrm{O} 2-\mathrm{C} 4-\mathrm{H} 4, \tau$, equal to $180^{\circ}$.

The molecule shows a first hydration shell with about 35 water molecules: of these only about 25 are constantly present, 18 are at a distance equal to lower than $2.5 \mathrm{~A}$ and only 5 have an interaction energy larger than $-21 \mathrm{~kJ} / \mathrm{mole}$.

The results do not support the hypothesis that the role of $\mathrm{O} 1$ in muscarinic activity is that of increasing hydrophylic bonds.
\end{abstract}

Key words: Muscarine solution, Monte Carlo simulation.

\section{Introduction}

In recent years, computer simulation of dilute solutions of biomolecules proved [1] to be a successful source of information for the energy profile and the hydration structure of the system.

In a previous paper [2], a Monte Carlo (MC) simulation of acetylcholine (Ach), a particularly important neurotransmitter, in water solution was carried out using interaction potentials derived from ab-initio computations.

The present paper reports the results obtained for $\mathrm{MC}$ simulations of Muscarine (Mu) in water solution based on the ab-initio potentials calculated in [3].

The monovalent cation $\mathrm{Mu}$ is also a very important neurotransmitter active on cholinergic receptors. The molecular nature and structural requirements for this type of activity are still under question [4] and various theoretical studies have been previously reported [5], but they were limited to the individual molecule, i.e. in vacuum, and by the use of approximate methods. Abinitio SCF-LCAO-MO computations on this molecule have indicated [3] that the positive charge is mainly distributed among the hydrogens of the three methyl and the methylene groups of the quaternary nitrogen: each methyl group carries about $27-30 \%$ of the charge, while the methylene group carries about $20 \%$; the nitrogen carries a negative charge. Thus, globally, $90 \%$ of the positive charge is symmetrically distributed on the quaternary globe.

Reprint requests to Prof. C. Margheritis, Department of Physical Chemistry, Viale Taramelli 16, 27100 Pavia, Italy.

\section{The Model}

The Mu cation was analyzed in the crystal structure given by Jellinek [6] for the Mu iodide which corresponds to the active form determined by Waser [7]. Since no experimental data were available for the position of the hydrogen atoms, standard values for their bond lengths and angles were assumed. Figure 1 reports the $x, y$-projection of the molecule along with the notation used in the present paper to identify the atoms. It should be noted that in this figure the dihedral angle $\mathrm{HO} 2-\mathrm{O} 2-\mathrm{C} 4-\mathrm{H} 4$ (angle between the two planes through $\mathrm{C} 4-\mathrm{O} 2$ ), $\tau$, has a value of $180^{\circ}$, even though the ab-initio analysis of the rotational barrier around the $\mathrm{C} 4-\mathrm{O} 2$ bond indicated [3] that in vacuum the preferred conformation has $\tau$ close to $0^{\circ}$ with a rotational barrier of about $25 \mathrm{~kJ} / \mathrm{mole}$.

Calculations were carried out using the MC method in the Metropolis version to simulate an NVT ensemble at $298 \mathrm{~K}$. Two different systems were studied, consisting of periodic cubes containing 502 mobile waters and a fixed $\mathrm{Mu}$ molecule having the conformation with $\tau=180^{\circ}$ (Musca) or that with $\tau=0^{\circ}$ (Muscato). The water molecules are initially in one of the optimal configurations for pure water at the same temperature. The minimum image periodic boundary condition has been applied to the water molecules in order to eliminate surface effects, but no solute-solute interaction has been included and thus the system represents an infinitely dilute aqueous solution. Solventsolvent interactions are described by the MCY potential [8], which (obtained using ab-initio compu-

0932-0784 / $90 / 0800-1009 \$ 01.30 / 0$. - Please order a reprint rather than making your own copy. 


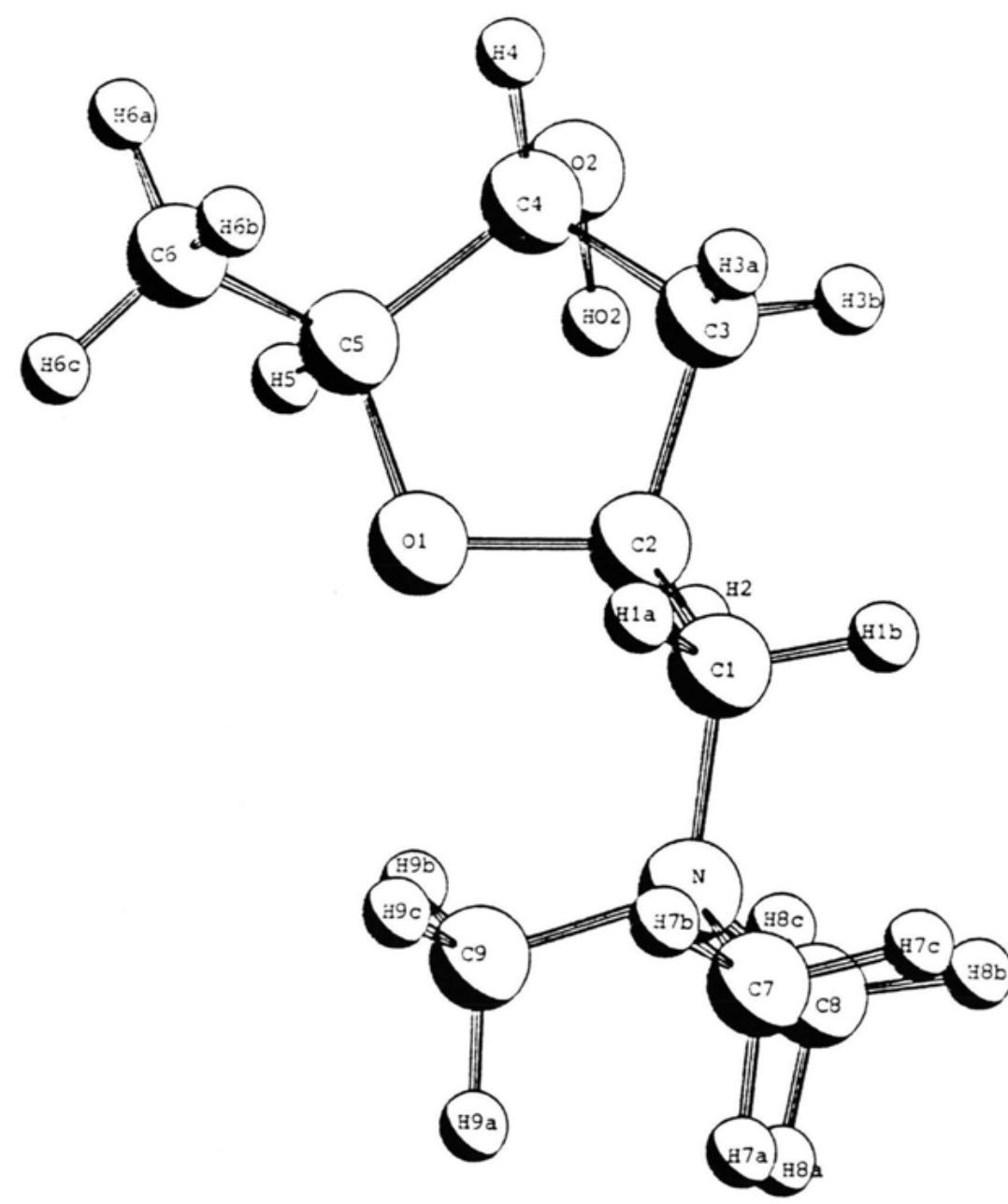

Fig. 1. $x, y$ projection of the muscarine molecule. tations with the Configuration Interaction method) is the most studied one and has been proved to give realistic results for the structure and static properties of liquid water. The same potential was also used for the simulations of [2], where further details can be found.

The solute-water interactions were described by the potentials evaluated in [3]. These were obtained through ab-initio SF-LCAO-MO computations using a $7 / 3$ basis set contracted to a minimal one [9] and the molecular program described in [10]. In essence, the $\mathrm{Mu}$-water system was studied at many (more than 500 ) different separations and orientations of the water molecule. The Mu-water interaction energies, corrected for the basis set superposition error with the counterpoise method [11], could then be calculated and fitted to the analytical form

$$
V=\sum_{i=1}^{n} \sum_{j=1}^{3} I_{i j},
$$

where $n$ is the number of atoms of $\mathrm{Mu}$, and $i$ and $j$ are the indices for the atom of $\mathrm{Mu}$ and water, respectively. The pair potential $I_{i j}$ has the form

$I_{i j}=-A_{i j}^{\mathrm{ab}}\left(r_{i j}\right)^{-6}+B_{i j}^{\mathrm{ab}}\left(r_{i j}\right)^{-12}+\mathrm{C}_{i j}^{\mathrm{ab}} q_{i} q_{j}\left(r_{i j}\right)^{-1}$,

where $q_{i}$ and $q_{j}$ are the net charges obtained from Mulliken population analysis for the atom $i$ and $j$, respectively, $A, B$, and $C$ are fitting constants and a and $\mathrm{b}$ are the class indices. A class is characterized by the electronic environment of the atom, different parameters being used to differentiate atoms with the same atomic number. Details for the class definition 
Table 1. Class and net charge (in fraction of electron) for the atoms of muscarine.

\begin{tabular}{llllll}
\hline Atom & Class & Charge & Atom & Class & Charge \\
\hline O1 & 1 & -0.4029 & H3b & 10 & 0.2745 \\
C2 & 2 & -0.0425 & H4 & 10 & 0.2724 \\
C3 & 3 & -0.5189 & H5 & 10 & 0.2547 \\
C4 & 2 & -0.0559 & H6a & 11 & 0.2829 \\
C5 & 2 & -0.0515 & H6b & 11 & 0.2567 \\
C6 & 4 & -0.7819 & H6c & 11 & 0.2752 \\
C1 & 5 & -0.3551 & HO2 & 12 & 0.3086 \\
O2 & 6 & -0.4504 & H7a & 13 & 0.3073 \\
N & 7 & -0.2671 & H7b & 13 & 0.3078 \\
C7 & 8 & -0.6117 & H7c & 13 & 0.3058 \\
C8 & 8 & -0.6217 & H8a & 13 & 0.3125 \\
C9 & 8 & -0.6269 & H8b & 13 & 0.3096 \\
H1a & 9 & 0.2997 & H8c & 13 & 0.3084 \\
H1b & 9 & 0.2933 & H9a & 13 & 0.2996 \\
H2 & 10 & 0.2177 & H9b & 13 & 0.3324 \\
H3a & 10 & 0.2583 & H9c & 13 & 0.3087 \\
\hline
\end{tabular}

and a discussion on the analytical form of the potential can be found in [12]. These potentials are limited by the fact that, owing to the size of the molecules involved, relatively small atomic orbital basis sets were used to calculate the interaction energies. Nevertheless, the use of the counterpoise corrections, as pointed out by Kolos [ $11 \mathrm{~b}$ ], significantly improves the results and, moreover, at present this is the only quantum-mechanically derived potential for muscarinewater interactions available for use in computer simulations. For the constants of the pair potentials used cf. Tables 1 and 2.

\section{Results}

For each system, $5 \times 10^{6}$ configurations were generated: the initial $3 \times 10^{6}$ steps were used for equilibration and the remaining $2 \times 10^{6}$ steps to evaluate the average properties of the system. In order to test the statistical significance of the results thus obtained, for each system the same calculations were also carried out starting from a different water configuration: the obtained deviations were within the statistical error. The satisfactory convergence of the calculations was also carefully verified. The final results are summarized in Table 3 where the solvent-solvent interaction energy per water molecule $\left(E_{\mathrm{ww}}\right)$ and the solute-solvent one $\left(E_{\mathrm{WM}}\right)$ are reported along with the total energy $\left(E_{\text {tot }}\right)$. As it can be noted, the Musca solution appears to be more stable than Muscato: thus in water solution the preferred conformation, on the basis of
Table 2. Pair potential coefficients for the system muscarinewater (the energy is in $\mathrm{kJ} / \mathrm{mole}$ if the distance is in $\AA$ and the $C$ coefficient is multiplied by 1389.3 ).

\begin{tabular}{rrll}
\hline Class & \multicolumn{1}{c}{$A_{i, \mathrm{O}}$} & $B_{i, \mathrm{o}}$ & $C_{i, \mathrm{o}}$ \\
\hline 1 & $-0.701770 \mathrm{D}+01$ & $0.392991 \mathrm{D}+06$ & $0.950000 \mathrm{D}+00$ \\
2 & $-0.148383 \mathrm{D}+02$ & $0.645010 \mathrm{D}+07$ & $0.103000 \mathrm{D}+01$ \\
3 & $-0.148360 \mathrm{D}+02$ & $0.557083 \mathrm{D}+07$ & $0.979859 \mathrm{D}+00$ \\
4 & $-0.137017 \mathrm{D}+01$ & $0.357558 \mathrm{D}+07$ & $0.978440 \mathrm{D}+00$ \\
5 & $0.531933 \mathrm{D}+01$ & $0.255502 \mathrm{D}+07$ & $0.103859 \mathrm{D}+01$ \\
6 & $0.841808 \mathrm{D}+04$ & $0.416495 \mathrm{D}+07$ & $0.979930 \mathrm{D}+00$ \\
7 & $0.384212 \mathrm{D}+05$ & $0.388890 \mathrm{D}+08$ & $0.978252 \mathrm{D}+00$ \\
8 & $-0.145005 \mathrm{D}+02$ & $0.998390 \mathrm{D}+06$ & $0.979887 \mathrm{D}+00$ \\
9 & $-0.566911 \mathrm{D}+01$ & $0.218455 \mathrm{D}+04$ & $0.102002 \mathrm{D}+01$ \\
10 & $-0.148570 \mathrm{D}+02$ & $0.363675 \mathrm{D}+04$ & $0.102012 \mathrm{D}+01$ \\
11 & $-0.148438 \mathrm{D}+02$ & $0.822545 \mathrm{D}+04$ & $0.978886 \mathrm{D}+00$ \\
12 & $0.593379 \mathrm{D}+03$ & $0.140766 \mathrm{D}+04$ & $0.100399 \mathrm{D}+01$ \\
13 & $-0.145633 \mathrm{D}+02$ & $0.467198 \mathrm{D}+04$ & $0.102013 \mathrm{D}+01$ \\
\hline $\mathrm{Class}$ & $A_{i, \mathrm{H}}$ & $B_{i, \mathrm{H}}$ & $C_{i, \mathrm{H}}$ \\
\hline 1 & $-0.148480 \mathrm{D}+02$ & $0.740660 \mathrm{D}+04$ & $0.975273 \mathrm{D}+00$ \\
2 & $-0.148597 \mathrm{D}+02$ & $0.227221 \mathrm{D}+06$ & $0.978497 \mathrm{D}+00$ \\
3 & $-0.148655 \mathrm{D}+02$ & $0.548121 \mathrm{D}+04$ & $0.100453 \mathrm{D}+01$ \\
4 & $-0.148910 \mathrm{D}+02$ & $0.891020 \mathrm{D}+05$ & $0.979757 \mathrm{D}+00$ \\
5 & $0.113860 \mathrm{D}+04$ & $0.133619 \mathrm{D}+04$ & $0.978927 \mathrm{D}+00$ \\
6 & $0.105363 \mathrm{D}+03$ & $0.133322 \mathrm{D}+04$ & $0.979736 \mathrm{D}+00$ \\
7 & $-0.871230 \mathrm{D}+04$ & $0.185757 \mathrm{D}+08$ & $0.103215 \mathrm{D}+01$ \\
8 & $-0.588245 \mathrm{D}+01$ & $0.223559 \mathrm{D}+04$ & $0.979889 \mathrm{D}+00$ \\
9 & $-0.225930 \mathrm{D}+01$ & $0.127275 \mathrm{D}+04$ & $0.102001 \mathrm{D}+01$ \\
10 & $-0.148551 \mathrm{D}+02$ & $0.555409 \mathrm{D}+04$ & $0.102009 \mathrm{D}+01$ \\
11 & $0.170577 \mathrm{D}+03$ & $0.160399 \mathrm{D}+05$ & $0.985915 \mathrm{D}+00$ \\
12 & $-0.148390 \mathrm{D}+02$ & $0.561585 \mathrm{D}+05$ & $0.102074 \mathrm{D}+01$ \\
13 & $0.676436 \mathrm{D}+01$ & $0.397081 \mathrm{D}+04$ & $0.102000 \mathrm{D}+01$ \\
\hline & & &
\end{tabular}

Table 3. Average interaction energies per water molecule (in $\mathrm{kJ} /$ mole) obtained from MC simulations.

\begin{tabular}{lll}
\hline & Musca & Muscato \\
\hline$E_{\mathrm{Ww}}$ & $-35.610 \pm 0.029$ & $-35.384 \pm 0.029$ \\
$E_{\mathrm{WM}}$ & $-0.866 \pm 0.012$ & $-0.933 \pm 0.012$ \\
$E_{\text {tot }}$ & $-36.476 \pm 0.029$ & $-36.321 \pm 0.029$ \\
\hline
\end{tabular}

purely enthalpic considerations, should be that with $\tau=180^{\circ}$. This, however, is not the more stable conformation if only the isolated molecule or the solute-solvent interaction energy were taken into account. It is the solvent that seems to re-arrange better with a larger solvent-solvent interaction energy.

The analysis of the structural environment of a simple liquid in a computer simulation is normally given by the radial distribution function (r.d.f.). Unfortunately, in the case of a complex polyatomic solute, the chemical meaning of the calculated functions is questionable. Attempts were made to overcome this problem [13], but no unambiguous solution has yet 

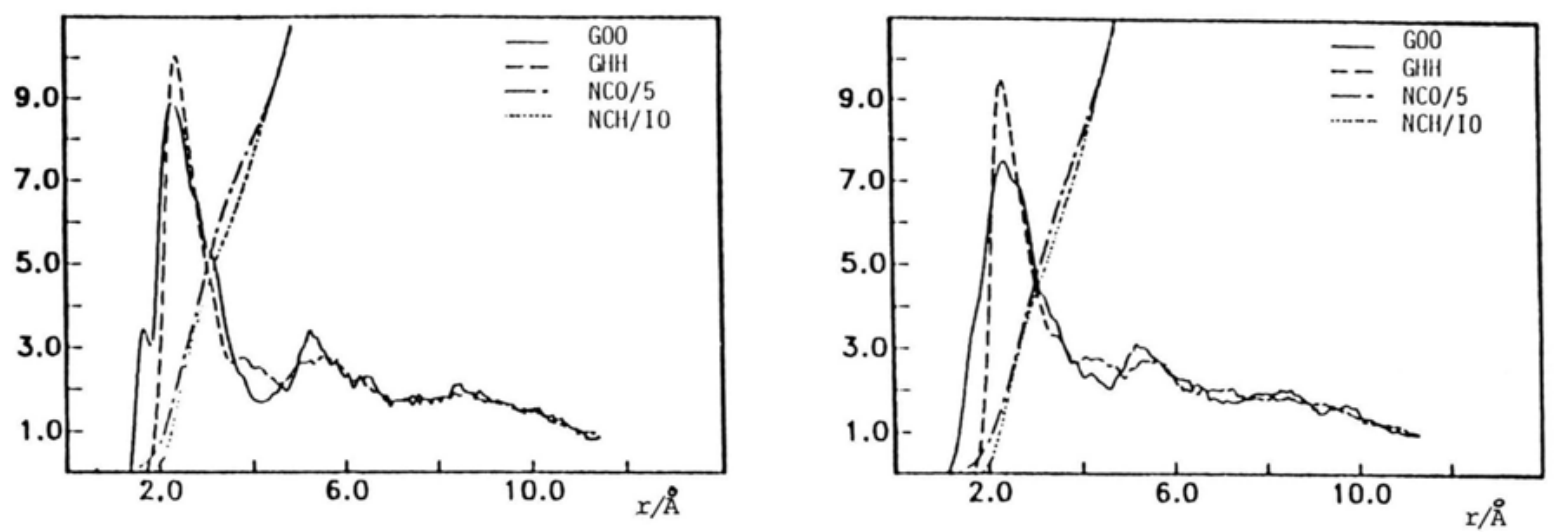

Fig. 2. Radial distribution functions relative to water oxygen $\left(G_{\mathrm{OO}}\right)$ and hydrogen $\left(G_{\mathrm{HH}}\right)$. The corresponding coordination numbers $\left(N_{\mathrm{CO}}\right.$ and $N_{\mathrm{CH}}$, respectively) up to $4.5 \AA$ are also reported. Left: $\tau=180^{\circ}$; right: $\tau=0^{\circ}$.

been proposed. Nevertheless, as indicated in [2], it was found useful, mainly because of the major role played by the almost spherical cationic head of the Mu molecule, to obtain through the MC history a "global r.d.f." calculated attributing the water oxygen or hydrogen to the closest $\mathrm{Mu}$ atom. Figure 2 reports the results obtained for the two solutions, along with the coordination number up to $4.5 \AA$. The calculated r.d.f.'s show a first minimum at about $4.1-4.3 \AA$ and a well structured second hydration shell.

Although the calculated functions for the two conformations appear slightly different, in both cases they indicate a first hydration shell (f.h.s.) containing about 35 water molecules. Moreover, in order to have a deeper view of the f.h.s., a different statistical analysis was performed by following the single water molecules during the MC chain: this shows that only about 25 of the water molecules in the f.h.s. are constantly present; 18 of these are at an average distance equal or lower than $2.5 \AA$, and only 5 have an interaction energy larger than $-21 \mathrm{~kJ} /$ mole. Taking into account that, for those interactions not exceeding $-21 \mathrm{~kJ} /$ mole, the temperature effect and the surrounding water molecules are important factors in determining the water orientation, particular attention was given to these 5 molecules.

Figures $3 \mathrm{a}$ and $4 \mathrm{a}$ show stereo views of the 5 strongly bound water molecules in a typical configuration for the Musca and for the Muscato systems, respectively. In Table 4 the calculated average interaction energies and distances along with the $\mathrm{Mu}$ atom involved in the interaction are reported. Although in both cases one water molecule interacts with the hy-
Table 4. Average interaction energies (in $\mathrm{kJ} / \mathrm{mole}$ ) and distances (in $\AA$ ) of the strongly bound water molecules.

\begin{tabular}{llll}
\hline Mol. Nr. & Energy & Distance & Mu atom \\
\hline Musca & & & \\
1 & -23.1 & 2.35 & $\mathrm{H} 9 \mathrm{c}$ \\
2 & -36.6 & 1.63 & $\mathrm{HO} 2$ \\
3 & -23.7 & 2.30 & $\mathrm{H} 1 \mathrm{~b}$ \\
4 & -25.8 & 2.41 & $\mathrm{H} 8 \mathrm{~b}$ \\
5 & -24.3 & 2.50 & $\mathrm{H} 7 \mathrm{a}$ \\
Muscato & & & \\
1 & -33.5 & 2.23 & $\mathrm{H} 1 \mathrm{a}$ \\
2 & -56.5 & 1.56 & $\mathrm{HO} 2$ \\
3 & -24.4 & 2.31 & $\mathrm{H} 8 \mathrm{c}$ \\
4 & -28.8 & 2.05 & $\mathrm{H} 8 \mathrm{~b}$ \\
5 & -28.2 & 2.49 & $\mathrm{H} 7 \mathrm{~b}$ \\
\hline
\end{tabular}

droxyl group and the other four with the cationic head (one with the methylene and the others with the methyl groups), the hydration profile of the two systems is in this respect differentiated and probably responsible for the different $E_{\mathrm{WM}}$ values obtained: the global interaction energy for these molecules is $-133.5 \mathrm{~kJ} / \mathrm{mole}$ in Musca and $-171.4 \mathrm{~kJ} / \mathrm{mole}$ in Muscato, thus the difference compares well with the about $34 \mathrm{~kJ} / \mathrm{mole}$ which differentiates the $E_{\mathrm{wm}}$ values calculated for the two systems. The only properly hydrogen bonded water molecule is the one interacting with $\mathrm{HO} 2$ (nr. 2 in Figs. $3 \mathrm{a}$ and $4 \mathrm{a}$ ), which is at a distance of about $1.6 \AA$. This water molecule in Muscato is practicaly fixed in the conformation shown in Fig. $4 \mathrm{a}$ with a very high interaction energy $(-56.5 \mathrm{~kJ} /$ mole), while in Musca it appears with a lower energy 
a
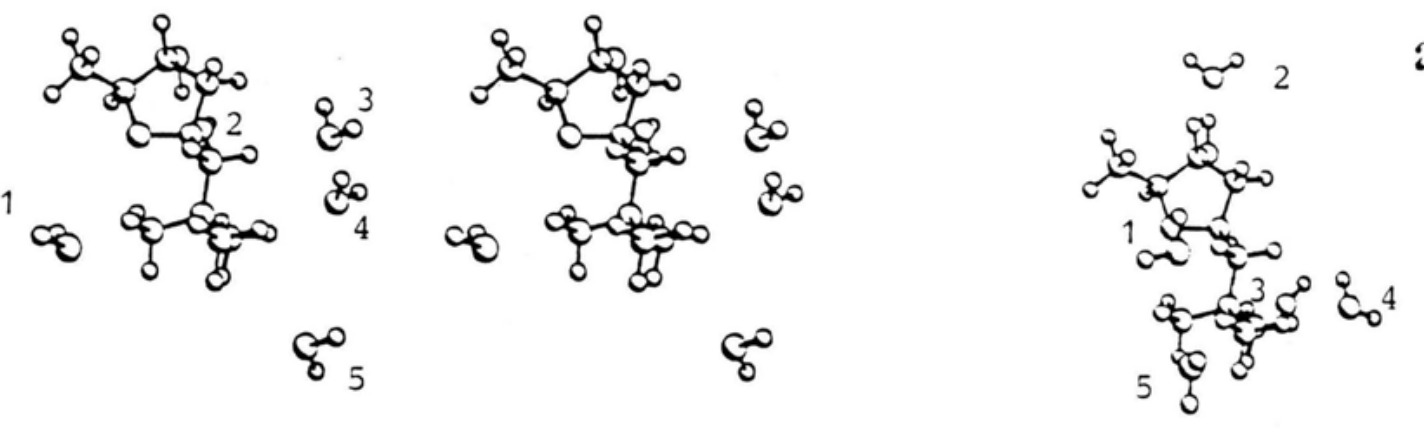

a

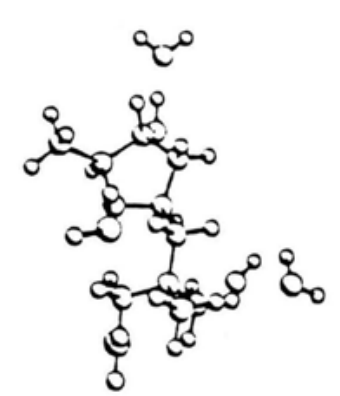

b
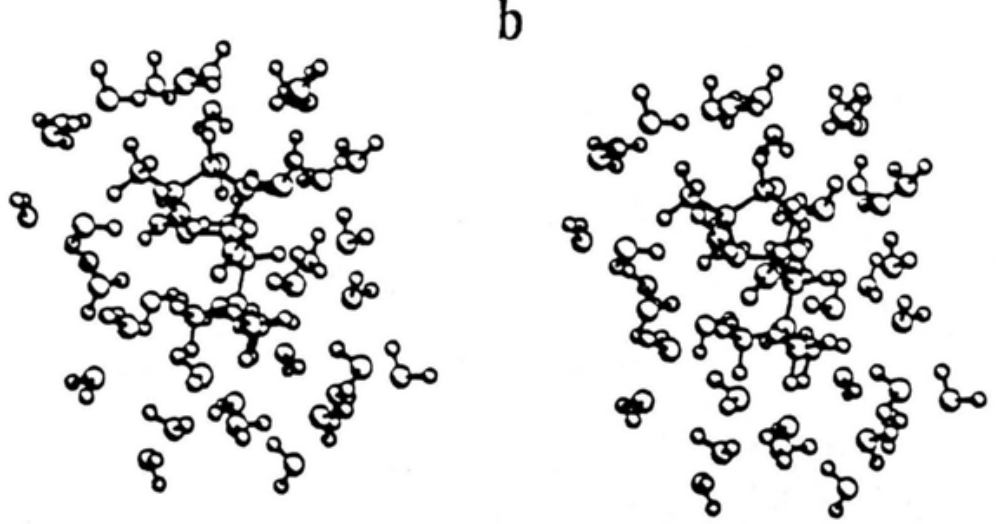

Fig. 3. Stereo view of the strongly bound water molecules (a) and of the first hydration shell (b) for the muscarine conformation with $\tau=180^{\circ}$.

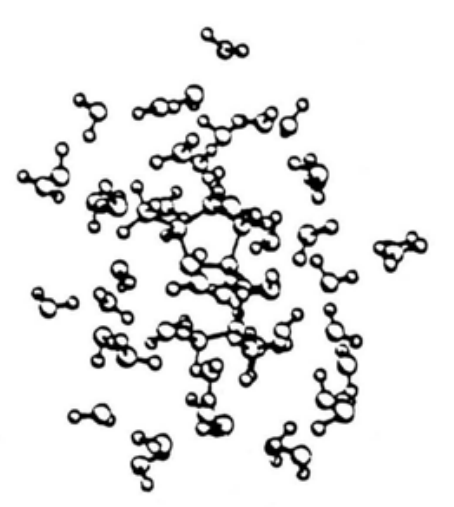

b

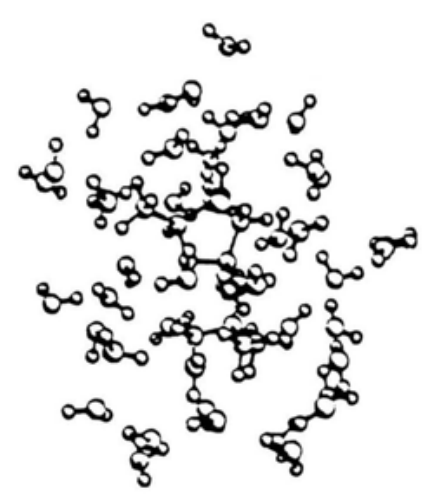

Fig. 4. Same as Fig. 3, for the muscarine conformation with $\tau=0^{\circ}$. 

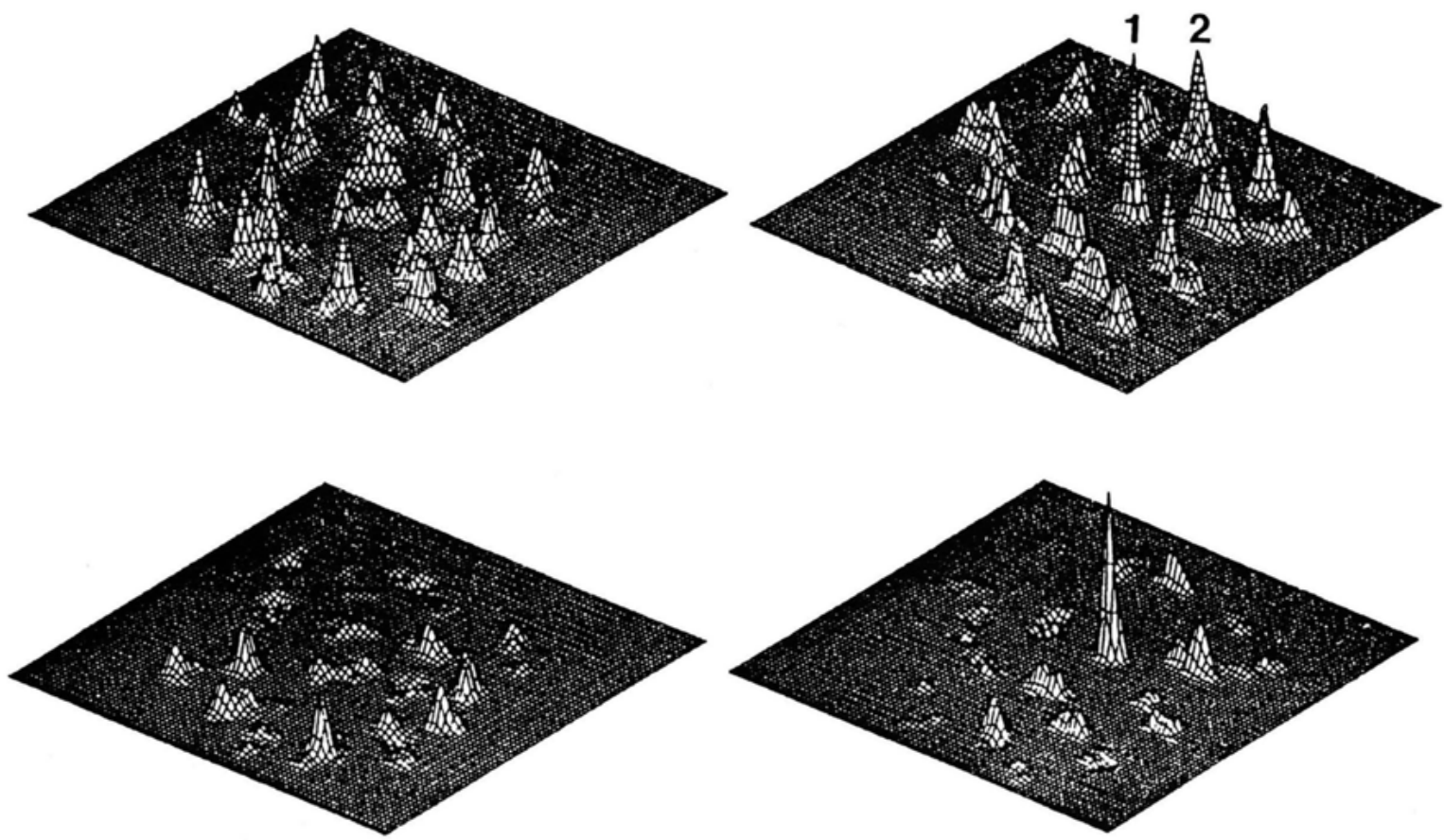

Fig. 5. Probability density maps for the oxygen (top) and for the average interaction energy (bottom) in the conformation with $\tau=180^{\circ}$ (for explanation, see text).

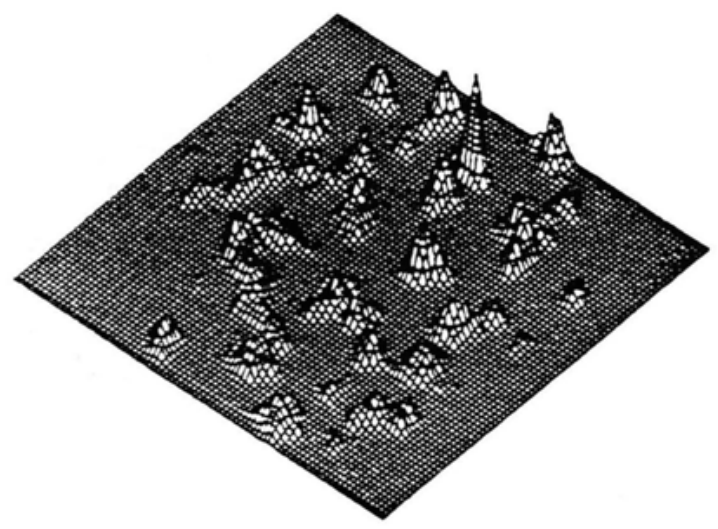

$(-36.6 \mathrm{~kJ} / \mathrm{mole})$ as an underneath bridge between $\mathrm{HO} 2$ and $\mathrm{H} 2$.

Figures $3 \mathrm{~b}$ and $4 \mathrm{~b}$ report stereo views of a typical f.h.s. configuration for the two systems.

Finally, by analyzing the MC history of the water molecules in the f.h.s., it was possible to obtain the probability density maps reported in Figs. 5 and 6. In Fig. 5 (concerning Musca) the left and right maps refer to the space with positive and negative $z$ coordinate, respectively. This subdivision was used in order to obtain a better separation of the contributions from the two hydrophilic groups of the molecule, since the cationic head is mainly in the positive $z$ space, while

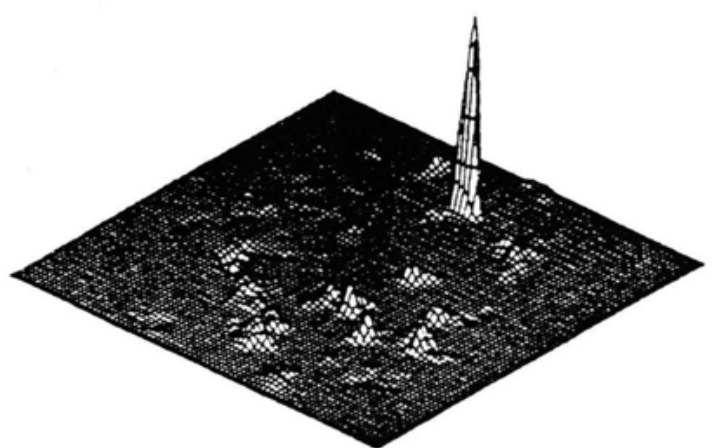

Fig. 6. Same as Fig. 5, for the conformation with $\tau=0^{\circ}$. the $\mathrm{OH}$ group is in the negative one. For each space section two maps are reported: the three-dimensional plot for the probability density of the water oxygen (top map) and that for the water-molecule interaction energy (bottom map). The analogous results obtained for the negative $z$ space in Muscato are shown in Fig. 6, whereas those for the positive $z$ space are not reported, since they are very similar to those for Musca. In this region the hydration of the cationic head is well evident and the energy map clearly shows the repulsive interaction energies (represented as holes) that characterize the almost hydrophobic ring. 
The plots obtained for the negative $z$ space underline the different behaviour of the two conformations studied: the oxygen density map calculated for Musca indicates that the hydroxyl group is hydrated both from outside the Mu molecule (peak nr. 2) and from underneath it (peak nr. 1), but while the former hydration gives a broad peak indicating a large space of accessibility, the latter gives a very sharp peak thus indicating that the water molecule is strongly localized. The sharp peak present in the energy probability plot (see right bottom map of Fig. 5) is related to this hydration location. In Muscato both the oxygen and the energy probability plots show only one high peak related to the hydroxyl hydration which is external.

\section{Conclusions}

Two main features should be underlined. The Mu molecule in water solution seems more stable in the conformation with $\tau=180^{\circ}$ than with $\tau=0^{\circ}$, even though the latter conformation is that with minimum energy in vacuum and, moreover, is the one with larger water-Mu interaction energy. It is the water-

[1] See e.g. E. Clementi (ed.), Modern Techniques in Computational Chemistry, ESCOM Science Publishers, Leiden 1989.

[2] C. Margheritis and G. Corongiu, J. Comp. Chem. 9, 1 (1988).

[3] C. Margheritis and G. Corongiu, Struct. Chem. 1, 385 (1990).

[4] R. Timoney, Int. J. Pharm. 15, 223 (1983).

[5] See e.g. a) L. Kier, Mol. Pharmacol. 3, 487 (1967). b) A. Liquori, A. Damiani, and J. De Cohen, J. Mol. Biol. 33, 445 (1968). - c) B. Pullman, Ph. Courriere, and J. Coubeils, Mol. Pharmacol. 7, 397 (1971). - d) R. Radna, D. Beveridge, and A. Bender, J. Amer. Chem. Soc. 95, (12), 3831 (1973).

[6] F. Jellinek, Acta Cryst. 10, 277 (1957).

[7] P. G. Waser, Pharmacol. Rev. 13, 465 (1961). water interaction energy that plays the major role in this case.

Calculations dealing with solvation of bio-molecules where the solvent is approximately represented by considering explicitly one solvent molecule only should be treated with great care. The present results clearly show that solvent-solvent interactions cannot be ignored.

As regards the biological activity of $\mathrm{Mu}$, it should be noted that the behaviour of $\mathrm{O} 1$, probably because of its interaction with the methyl hydrogen, does not support the hypotheses that its role in muscarinic activity consists in increasing hydrophylic bonds: the ring itself appears to be relatively hydrophobic and no water molecule was found in the proximity of $\mathrm{O} 1$ with negative interaction energy.

\section{Acknowledgement}

The work was partially carried out with the support of a CNR grant at the IBM Research Center in Kingston, N.Y., USA, directed by Prof. E. Clementi. The author acknowledges Dr. G. Corongiu for interesting discussions.

[8] O. Matsuoka, E. Clementi, and M. Yoshimine, J. Chem. Phys. 64, 1351 (1976).

[9] L. Gianolio, R. Pavani, and E. Clementi, Gazz. Chim. It. 108, 181 (1978).

[10] R. Gomperts and E. Clementi, KGNMOL: Documentation and user's guide for QCPE Program N. 538, QCPE Bull. 8, 33 (1988).

[11] a) S. F. Boys and F. Bernardi, Mol. Phys. 19, 553 (1970). b) W. Kolos, Theor. Chim. Acta 51, 219 (1979).

[12] E. Clementi, Computational Aspects for Large Chemical Systems, Springer-Verlag (Lecture Notes in Chemistry, Nr. 19), Berlin 1981.

[13] See e.g. M. Mezei, P. Mehrotra, and D. Beveridge, J. Biomol. Struct. Dynam. 2, 1 (1984).

[14] R. S. Givens and D. R. Rademacher, J. Med. Chem. 17, 457 (1974). 